\title{
Darwin-an experimental astronomy mission to search for extrasolar planets
}

\author{
Charles S. Cockell • Tom Herbst • Alain Léger • O. Absil •
}

Charles Beichman • Willy Benz • Andre Brack • Bruno Chazelas •

Alain Chelli • Hervé Cottin • Vincent Coudé du Foresto • William Danchi • Denis Defrère · Jan-Willem den Herder • Carlos Eiroa • Malcolm Fridlund •

Thomas Henning • Kenneth Johnston • Lisa Kaltenegger • Lucas Labadie •

Helmut Lammer • Ralf Launhardt • Peter Lawson • Oliver P. Lay •

Rene' Liseau • Stefan R. Martin • Dimitri Mawet • Denis Mourard •

Claire Moutou • Laurent Mugnier • Francesco Paresce •

Andreas Quirrenbach • Yves Rabbia • Huub J. A. Rottgering •

Daniel Rouan • Nuno Santos • Franck Selsis • Eugene Serabyn •

Frances Westall • Glenn White • Marc Ollivier • Pascale Bordé

Received: 26 October 2007 / Accepted: 6 August 2008 / Published online: 10 September 2008

(C) The Author(s) 2008. This article is published with open access at Springerlink.com

C. S. Cockell $(\bowtie)$

Planetary and Space Sciences Research Institute,

The Open University, Milton Keynes, MK7 6AA, UK

e-mail: c.s.cockell@open.ac.uk

T. Herbst $\cdot$ T. Henning $\cdot$ L. Labadie $\cdot$ R. Launhardt

Max Planck Institute fur Astronomie, Konigstuhl, 17,

69117 Heidelberg, Germany

A. Léger · B. Chazelas · M. Ollivier · P. Bordé

IAS, bat 121, Université Paris-Sud, F-91405 Orsay, France

O. Absil

Laboratoire d'Astrophysique de l'Observatoire de Grenoble,

414 rue de la Piscine, 38400 Saint Martin d'Hères, France

C. Beichman

Michelson Science Center, California Institute of Technology,

Pasedena, CA 91125, USA

W. Benz

Physikalisches Institut, University of Berne, Bern, Switzerland

A. Brack · F. Westall

Centre de Biophysique Moleculaire, CNPS,

Rue Charles Sadron, 45071 Orleans cedex 2, France 
Abstract As a response to ESA call for mission concepts for its Cosmic Vision 2015-2025 plan, we propose a mission called Darwin. Its primary goal is the study of terrestrial extrasolar planets and the search for life on them. In this paper, we describe different characteristics of the instrument.

Keywords Interferometer • Nulling interferometry •

Direct imaging of exoplanets $\cdot$ Exoplanets $\cdot$ Habitable zone

\section{The Darwin mission goals}

The discovery of extra-solar planets is one of the greatest achievements of modern astronomy. There are now more than 200 such objects known, and the recent detection of planets with masses approximately five times that of Earth demonstrates that extra-solar planets of low mass exist. In addition to providing a wealth of scientific information on the formation and structure of planetary systems, these discoveries capture the interest of both scientists

\footnotetext{
A. Chelli

Laboratoire d'Astrophysique de Grenoble (LAOG),

BP 53, 38041 Grenoble Cedex 9, France
}

H. Cottin

Laboratoire Interuniversitaire des Systèmes Atmosphériques Universités Paris 12, Paris 7 ,

CNRS UMR 7583 91, av. Di Général de Gaulle, 94010 Créteil Cedex, France

V. Coudé du Foresto · D. Rouan

LESIA-PHASE-Observatoire de Paris, 5 place Jules Janssen, 92190 Meudon, France

W. Danchi

Astrophysics Science Division, NASA Goddard Space Flight Center,

Greenbelt, MD 20771, USA

D. Defrère

Institut d'Astrophysique et de Géophysique de Liège,

17 Allée du 6 Août, 4000 Liège, France

J.-W. den Herder

SRON Netherlands Institute for Space Research,

Sorbonnelaan 2, 3584 CA Utrecht, The Netherlands

C. Eiroa

Dpto Fisica Toerica C-XI, Facultad de Ciencas, Universidad Autonoma de Madrid,

Cantoblanco, 28049 Madrid, Spain

M. Fridlund

Astrophysics Mission Division, European Space Agency, ESTEC, SCI-SA PO Box 299,

Keplerlaan 1 NL, 2200AG Noordwijk, The Netherlands

K. Johnston

United States Naval Observatory, 3450 Massachusetts Avenue NW,

Washington DC 20392, USA 
and the wider public with the profound prospect of the search for life in the Universe.

We propose an L-type mission, called Darwin, whose primary goal is the study of terrestrial extrasolar planets and the search for life on them. By its very nature, Darwin advances the first Grand Theme of ESA's Cosmic Vision. Accomplishing the mission objectives will require collaborative science across disciplines ranging from planet formation and atmospheres to chemistry and biology, and these disciplines will reap rewards from their contributions to the Darwin mission.

Darwin is designed to detect rocky planets similar to the Earth and perform spectroscopic analysis of them at mid-infrared wavelengths (6 to $20 \mu \mathrm{m})$, where the most advantageous contrast ratio between star and planet occurs. The spectroscopy will characterize the physical and chemical state of the planetary atmospheres and search for evidence of biological activity.

\section{Kaltenegger}

Harvard-Smithsonian Center for Astrophysics,

60 Garden St. MS20, Cambridge, MA 02138, USA

\section{H. Lammer}

Space Research Institute, Austrian Academy of Sciences,

Schmiedlstr. 6, 8042 Graz, Austria

P. Lawson · O. P. Lay · S. R. Martin · D. Mawet · E. Serabyn

Jet Propulsion Laboratory, California Institute of Technology,

4800 Oak Grove Drive, Pasadena, CA 91109, USA

R. Liseau

Onsala Space Observatory, Chalmers University of Technology,

SE-439 92 Onsala, Sweden

D. Mourard

Observatoire de la Côte d'Azur, Anevue Copernic, 06130 Grasse, France

C. Moutou

Laboratoire d'Astrophysique de Marseille (LAM), CNRS, Traverse du Siphon,

BP 8, Les Trois Lucs, 13376 Marseille cedex 12, France

L. Mugnier

ONERA/DOTA, B.P. 72, 92322 Châtillon cedex, France

F. Paresce

IASF-Bologna, INAF, Bologna, Italy

A. Quirrenbach

ZAH, Landerssternwarte, Koenigstuhl, 69117 Heidelberg, Germany

Y. Rabbia

Observatoire de la Cote d'Azur, Dpt GEMINI UMR CNRS 6203,

Av Copernic, 06130 Grasse, France 


\section{The Darwin mission profile}

\subsection{Baseline mission scope}

The Darwin mission consists of two phases, search and spectral characterization, whose relative duration can be adjusted to optimize scientific return. During the search phase of the mission (nominally 2 years), the mission will examine nearby stars for evidence of terrestrial planets in their habitable zone (HZ). An identified planet should be observed at least three times during the mission in order to characterize its orbit. The number of stars that can be searched depends on the level of zodiacal light in the system and the diameter of the collector telescopes. As a baseline, we estimate this number under the assumption of a mean exozodiacal density three times that in the solar system and collecting diameters of $2 \mathrm{~m}$. Over 200 stars can be screened under these conditions (Section 3.3.3). The mission focuses on solar type stars, including the F, G, K and some M spectral types.

The number of expected planetary detections depends upon the mean number of terrestrial planets in the HZ, per star, $\eta_{\text {Earth }}$. Our present understanding of terrestrial planet formation (Section 2.3) and our solar system, where there are two such planets (Earth and Mars) and one close to the HZ (Venus), point to a fairly high abundance of terrestrial planets. We assume hereafter that $\eta_{\text {Earth }}=1$. The COROT mission should reveal the abundance of small hot planets, and Kepler will evaluate $\eta_{\text {Earth }}$ as well as the size distribution of these objects several years before Darwin flies. These inputs will allow refinement of Darwin's observing strategy well in advance of launch.

During the characterization phase of the mission (nominally 3 years), Darwin will acquire spectra of each detected planet at a resolution of 20 and with sufficient signal-to-noise to measure the equivalent widths of $\mathrm{CO}_{2}, \mathrm{H}_{2} \mathrm{O}$, and $\mathrm{O}_{3}$ with a precision of $20 \%$ if they are in abundances similar to those in the Earth's atmosphere.

\section{H. J. A. Rottgering}

Leiden Observatory, Leiden University, PO Box 9513, 2300 RA Leiden, The Netherlands

\section{N. Santos}

Centro de Astrofisica, Universidade do Porto, Rua das Estrelas, 4150-762 Porto, Portugal

F. Selsis

CRAL (CNRS UMR 5574), Université de Lyon, Ecole Supérieure de Lyon, 46 Allée d'Italie, 69007 Lyon, France

G. White

Department of Physics and Astronomy, The Open University, Walton Hall, Milton Keynes MK7 6AA, UK

G. White

Space Science and Technology Department, CCLRC Rutherford Appleton Laboratory, Chilton, Didcot, Oxfordshire OX11 0QX, UK 
Spectroscopy is more time consuming than detection. With $\eta_{\text {Earth }}=1$, only a fraction of the detected planets can be studied spectroscopically. As shown in Section 3.3.3 for Earth-sized planets, Darwin can perform spectroscopy of $\mathrm{CO}_{2}$ and $\mathrm{O}_{3}$ on about 50 planets and of $\mathrm{H}_{2} \mathrm{O}$ on about 25 planets during the nominal 3-year characterization phase. Note that the mission profile retains flexibility, and optimization of the spectroscopy phase will be possible based on early results from the detection phase.

The general astrophysics program, if adopted, will comprise $10 \%$ to $20 \%$ of the mission time.

The primary science segment would then be reduced accordingly, with limited impact on its outcome.

\subsection{Extended mission scope}

An extension of the mission to 10 years will depend on the results gathered during the first 5 years. Such an extension could be valuable to observe more M stars, only $10 \%$ of the baseline time is attributed to them, search for big planets around a significantly larger sample of stars, and carry out additional measurements on the most interesting targets already studied.

\subsection{Darwin target catalogue}

The Darwin target star catalogue was generated from the Hipparcos catalogue by examining the distance $(<25 \mathrm{pc})$, brightness $(<12 \mathrm{~V}$-mag), spectral type (F, G, K, M main sequence stars), and multiplicity (no companion within 1 arcsec). The catalogue has considered different interferometer architectures, since they have different sky access (see Section 3.2). The Emma design can observe $99 \%$ of the sky (Section 3.2.1). The corresponding star catalogue contains 384 targets excluding M stars, and 625 stars including them. Figure 1 shows some features of these stars.
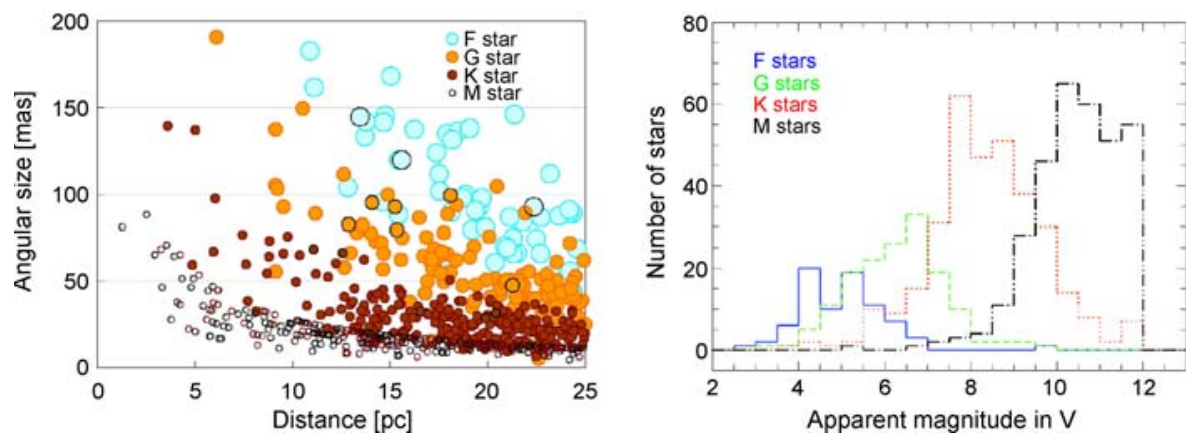

Fig. 1 Left, size of the habitable zone for the different spectral types of Darwin targets [3]. Right, histogram of their apparent visible magnitudes 


\section{Mission design}

\subsection{The Darwin concept and its evolution}

In order to disentangle the faint emission of an Earth-like planet from the overwhelming flux of its host star, the planetary system needs to be spatially resolved. This, in turn, requires an instrument up to $100 \mathrm{~m}$ in diameter when operating at mid-IR wavelengths since the angular size of the habitable zones around Darwin target stars ranges between 10 and 100 mas. A monolithic telescope of this size is presently not feasible, particularly since the observatory must be space-borne and cooled to provide continuous coverage and sensitivity between 6 and $20 \mu \mathrm{m}$.

As a result, interferometry has been identified as the best-suited technique to achieve mid-IR spectroscopy of Earth-like planets around nearby stars. In his pioneering paper, Bracewell [1] suggested that applying a $\pi$ phase shift between the light collected by two telescopes could be used to cancel out the on-axis star, while allowing the signal from an off-axis planet to pass through (Fig. 2). This technique, referred to as nulling interferometry, has been at the heart of the Darwin concept since its origin $[10,11]$ and many improvements have been studied since that date.

In addition to the planetary flux, a number of spurious sources contribute to the signal at the destructive output of the Bracewell interferometer:

- Residual star light, referred to as stellar leakage, caused by the finite size of the stellar photosphere and by imperfect efficiency of the interferometer;

- The local zodiacal background, produced by the disk of warm dust particles that surround our Sun and radiate at infrared wavelengths;

- The exozodiacal light, arising from the dust disk around the target star;

- The instrumental background produced by thermal emission within the instrument.
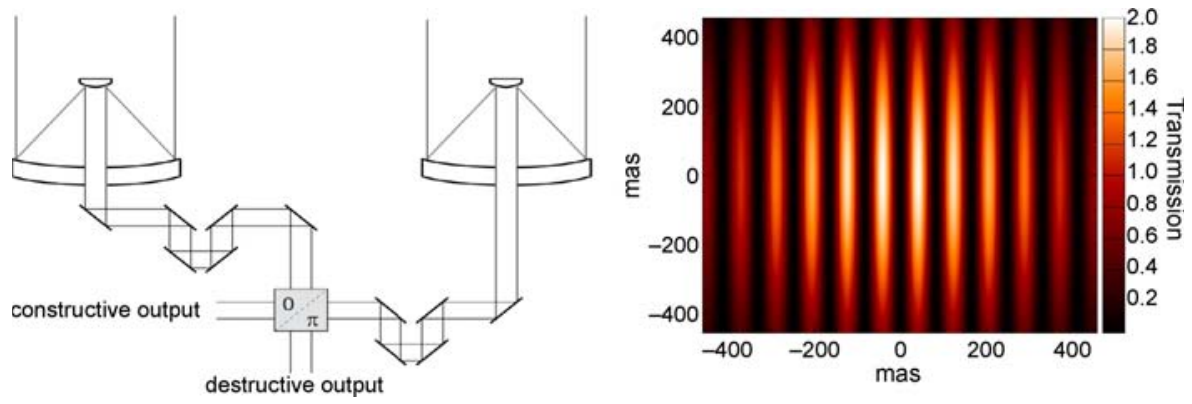

Fig. 2 Left, principle of a two-telescope Bracewell nulling interferometer. Right, associated transmission map, displayed for $\lambda=10 \mu \mathrm{m}$ and a $25-\mathrm{m}$ baseline array. This fringe pattern is effectively projected on the sky, blocking some regions while transmitting others 
Bracewell's original suggestion of rotating the array of telescopes can help disentangle the various contributions. The planet signal would then be temporally modulated by alternatively crossing high and low transmission regions, while the stellar signal and the background emission remain constant (except for the exozodiacal emission). Unfortunately, this level of modulation is not sufficient to achieve Darwin's goals, prompting a series of improvements to the strategy, including:

- Breaking the symmetry of the array to cancel all centro-symmetric sources, including the stellar leakage, the local and exozodiacal emissions;

- Performing faster modulation of the planet signal via internal modulation between the outputs of sub-interferometers

Merging of these two ideas has led to the concept of phase chopping [12] which is now regarded as a mandatory feature in space-based nulling interferometry. Figure 3 illustrates the principle. The outputs of two Bracewell interferometers are combined with opposite phase shifts $( \pm \pi / 2)$ to produce two "chopped states," which are mirrored with respect to the optical axis. Taking the difference of the photon rates obtained in the two chopped states gives the chopped response of the array, represented by the modulation map. This chopping process removes all centro-symmetric sources, including the stellar leakage and the exozodiacal emission.

Because the modulation efficiency varies across the field-of-view, the planet can only be localised and characterised through an additional level of modulation, provided by array rotation with a typical period of one day. The

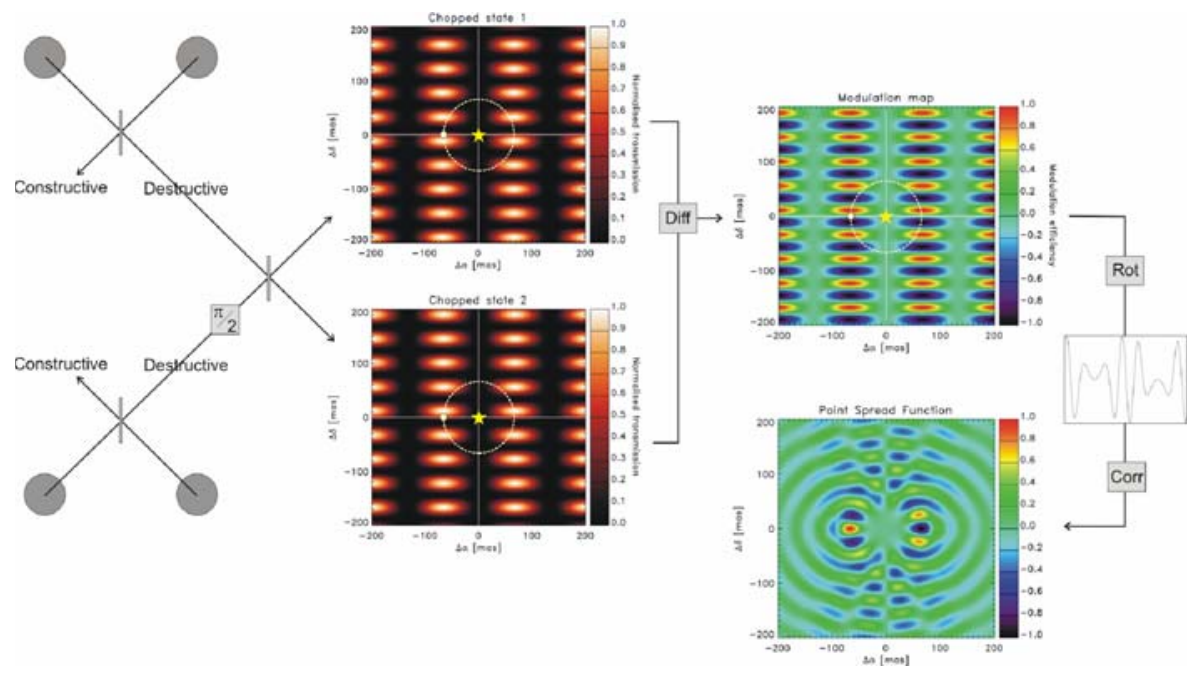

Fig. 3 Phase chopping for the X-array, a four-element rectangular configuration of telescopes (see Section 4.2). Combining the beams with different phases produces two conjugated chopped states, which are used to extract the planetary signal from the background. Array rotation then locates the planet by cross-correlation of the modulated chopped signal with a template 
variation of the chopped planet photon rate with the rotation angle of the array appears at the extreme right of Figure 3. These data must be inverted to obtain the fluxes and locations of any planets that are present. The most common approach is correlation mapping, which is closely related to the Fourier transform used for standard image synthesis. The result is a correlation map, displayed for a single point source in the low right part of the figure. This represents the point spread function (PSF) of the array.

This process, illustrated here for a single wavelength, is repeated across the waveband, and the maps are co-added to obtain the net correlation map. The broad range of wavelengths planned for Darwin greatly extends the spatial frequency coverage of the array, suppressing the side lobes of the PSF (Section 3.3.4).

A dozen array configurations using phase chopping have been proposed and studied at ESA and NASA during the past decade. In 2004, the two agencies agreed on common figures of merit to evaluate their performance. The most important criteria are the modulation efficiency of the beam combination scheme, the structure of the PSF and its associated ability to handle multiple planets, the overall complexity of beam routing and combination, and finally, the number of stars that can be surveyed during the mission lifetime (Section 3.3.3). Among the many configurations studied, the X-array has been identified as the most promising for the Darwin mission.

\subsection{Mission architecture}

The desire for maximum mission efficiency, technical simplicity, and the ability to detect multiple planets around as many stars as possible has guided the selection of mission architecture. Additional top-level requirements include:

- Two observing modes: nulling for extrasolar planet detection and spectroscopy, and constructive imaging for general astrophysics;

- Placement at L2 for passive cooling and low ambient forces;

- Launch with a single Ariane 5 rocket or two Soyuz-ST/Fregat vehicles;

- The ability to search at least 225 candidate stars with an exozodiacal background of one zodi, ${ }^{1}$ or 150 stars with an exozodiacal background of 10 zodis;

- Detection and measurement of terrestrial atmosphere biosignatures as described in Section 2.2 for at least 25 stars (with one zodi) or 15 stars (with 10 zodis; 2 m collectors);

- Time allocation of search as follows: G stars 50\%, K stars 30\%, F and M stars $10 \%$ each.

The effort to turn these requirements into a workable mission culminated in 2005-2006 with two parallel assessment studies of the Darwin mission. Two

\footnotetext{
${ }^{1}$ A "zodi" is defined as the density of our local zodiacal dust disk and acts as a scaling factor for the integrated brightness of exozodiacal dust disks.
} 
array architectures have been thoroughly investigated during these studies: the four-telescope X-array and the three-telescope TTN. These studies included the launch requirements, payload spacecraft, and the ground segment during which the actual mission science would be executed. Almost simultaneously, NASA/JPL initiated a similar study in the context of the Terrestrial Planet Finder Interferometer (TPF-I).

These efforts on both sides of the Atlantic have resulted in a convergence and consensus on mission architecture. The baseline for Darwin is a noncoplanar, or Emma ${ }^{2}$-type X-array, with four collector spacecraft (CS) and a single beam combiner spacecraft (BCS). This process also identified a back-up option, in case unforeseen technical obstacles appear: a planar X-array.

\subsubsection{The Emma X-array architecture}

Figure 4 shows the non-coplanar Emma X-array. Four simple collector spacecrafts fly in a rectangular formation and feed light to the beam combiner spacecraft located approximately $1,200 \mathrm{~m}$ above the array. This arrangement allows baselines up to $170 \mathrm{~m}$ for nulling measurements and up to $500 \mathrm{~m}$ for the general astrophysics program.

The $\mathrm{X}$-array configuration separates the nulling and imaging functions, thus allowing independent optimal tuning of the shorter dimension of the array for starlight suppression and that of the longer dimension for resolving the planet. Most other configurations are partially degenerate for these functions. The Xarray also lends itself naturally to techniques for removing instability noise, a key limit to the sensitivity of Darwin (Section 3.3.2). The assessment studies settled on an imaging to nulling baseline ratio of 3:1, based on scientific and instrument design constraints. A somewhat larger ratio of 6:1 may improve performance by simplifying noise reduction in the post-processing of science images (Section 3.3.2).

Each of the collector spacecraft (CS) contains a spherical mirror and no additional science-path optics (some components may be needed for configuration control). The four CS fly in formation to synthesize part of a larger paraboloid-the Emma configuration is a single, sparsely filled aperture. Flexing of the CS primary mirrors or deformable optics within the beam combiner spacecraft will conform the individual spheres to the larger paraboloid.

The beam combiner spacecraft (BCS) flies near the focal point of this synthesized paraboloid. Beam combination takes place on a series of optical benches arranged within the BCS envelope. The necessary optical processing includes:

- Transfer optics and BCS/CS metrology;

- Correction and modulation, including optical delay lines, tip-tilt, deformable mirrors;

\footnotetext{
${ }^{2}$ Emma was Charles Darwin's wife. The credit of the X-array is due to O. Lay and S. Dubovitsky [9], and that of the Emma configuration to A. Karlsson [4] and Thales Alenia Space [17].
} 


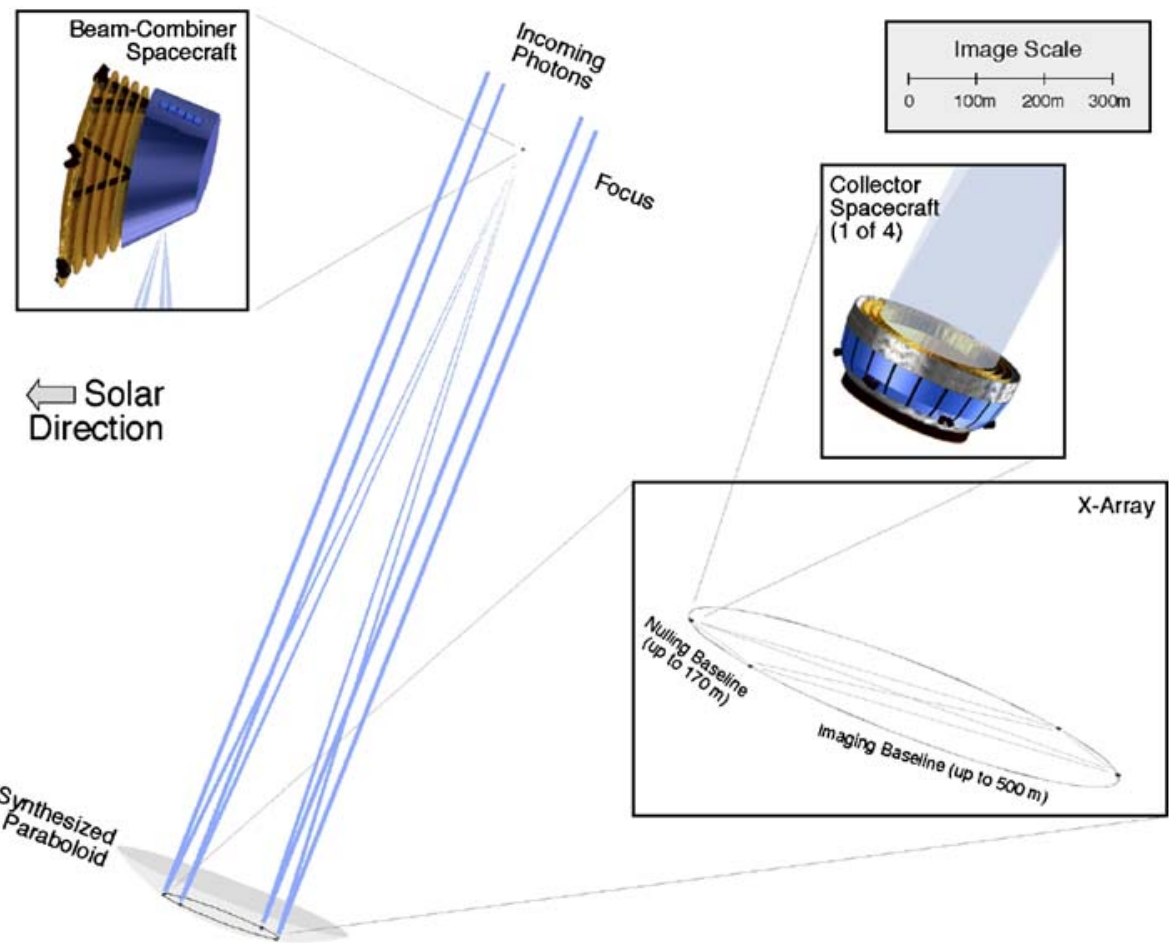

Fig. 4 The Emma X-array configuration consists of four collector spacecrafts and a beam combiner spacecraft. Spherical mirrors in the collectors form part of a large, synthetic paraboloid, feeding light to the beam combiner at its focus

- Mirrors, wavefront sensors, and beam switching;

- Possibly, spectral separation to feed the science photons into two separate channels;

- Phase shifting, beam mixing;

- Recombination, spectral dispersion and detection

The collector and beam combiner spacecrafts use sunshades for passive cooling to $<50 \mathrm{~K}$.

An additional refrigerator within the BCS cools the detector assembly to below $10 \mathrm{~K}$.

Due to the configuration of the array and the need for solar avoidance, the instantaneous sky access is limited to an annulus with inner and outer halfangles of $46^{\circ}$ and $83^{\circ}$ centred on the anti-Sun vector (Fig. 5). This annulus transits the entire ecliptic circle during one year, hence giving access to almost the entire sky (99\%).

For launch, the collector and beam-combiner spacecraft are stacked within the fairing of an Ariane 5 ECA vehicle. Total mass (Section 6) is significantly less than 6.6 tons, the launcher capability. Table 1 lists key parameters of 
Fig. 5 At any given time, the Emma X-Array can observe an annular region on the sky between $46^{\circ}$ and $83^{\circ}$ from anti-Sun direction. During one Earth year, this annulus executes a complete circle, giving access to $99 \%$ of the celestial sphere

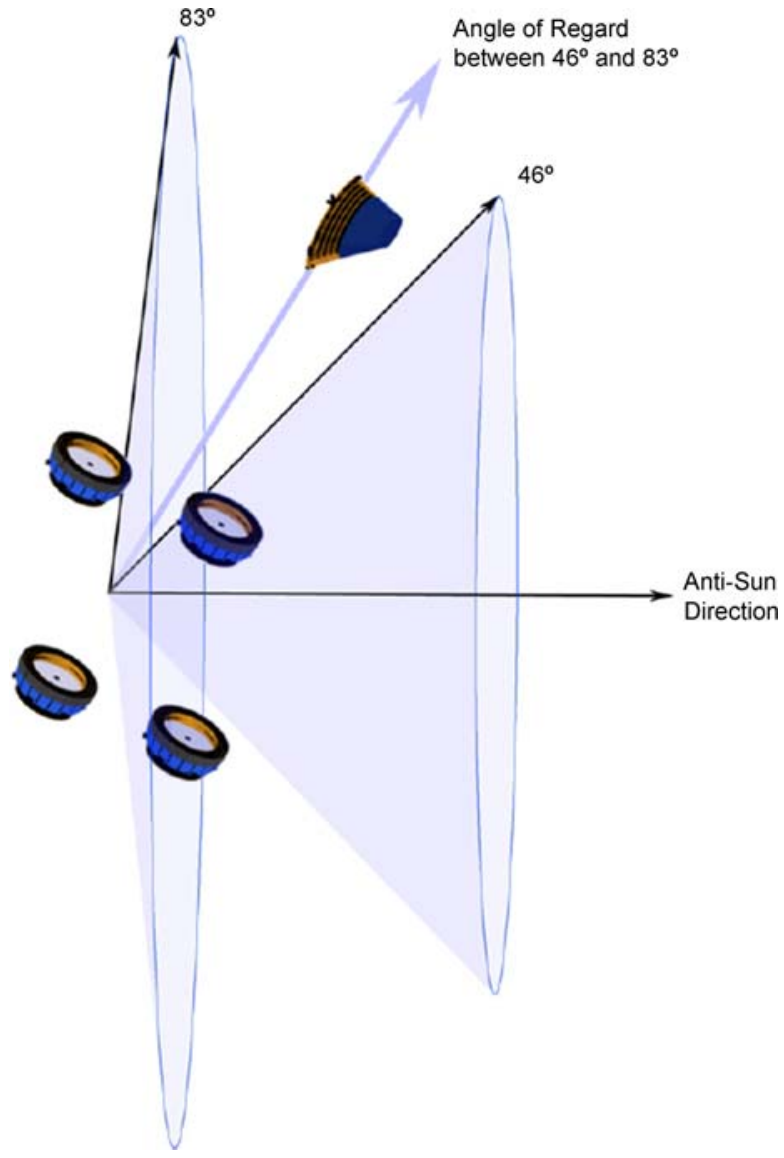

the Darwin Emma X-array. These values represent the results of the various assessment and system level studies conducted by ESA and NASA.

\subsection{Mission performance}

\subsubsection{Detecting earths}

Darwin's instruments will encounter a number of extraneous signals (see Fig. 6). The planetary flux must be extracted and analysed in the presence of these other components. The discrimination is performed by nulling the stellar light as much as possible, and by appropriate modulation (Section 3.1) that produces a zero mean value for the different background sources. Unfortunately, modulation cannot eliminate the quantum noise (sometimes referred to as photon noise) associated with these sources.

For a given integration time, the signal is proportional to the number of planetary photons, and the quantum noise increases with the square root of the number of extraneous photons (Fig. 6). As described in the next section, 
Table 1 Key Darwin parameters

\begin{tabular}{|c|c|}
\hline Item & Value or comment \\
\hline Collector spacecraft (CS) & 4 free-flyers, passively cooled to $<50 \mathrm{~K}$ \\
\hline CS optics & Lightweight spherical mirrors, diameter about $2.0 \mathrm{~m}$, no deployables \\
\hline CS array configuration & $\mathrm{X}$-array with aspect ratio $3: 1-6: 1$ (to be optimized) \\
\hline Available baselines & 7 to $168 \mathrm{~m}$ nulling, 20 to $500 \mathrm{~m}$ imaging option \\
\hline Beam combiner $(\mathrm{BCS})$ & 1 free flying spacecraft, passively cooled to $<50 \mathrm{~K}$ \\
\hline Beam combiner optics & Transfer, modulation, beam-mixing, recombination, spectroscopy \\
\hline Detection & $\begin{array}{l}\text { Mid-IR detector about } 500 \times 8 \text { pixels for nulling, } \\
(300 \times 300 \text { for imaging option }), \text { cooled to }<10 \mathrm{~K}\end{array}$ \\
\hline Detector cooling & Low vibration refrigerator, e.g. absorption coolers \\
\hline Telemetry & Require about $1 \mathrm{GBit} / \mathrm{s}$, direct downlink from BCS \\
\hline Operating wavelength & 6-20 $\mu \mathrm{m}$, includes $\mathrm{H}_{2} \mathrm{O}, \mathrm{O}_{3}, \mathrm{CH}_{4}, \mathrm{CO}_{2}$ signatures \\
\hline Field of view & Typically 1 arcsec at $10 \mu \mathrm{m}$ \\
\hline Null depth & $10^{-5}$, stable over $\sim 5$ days \\
\hline Angular resolution & 5 milliarcsec at $10 \mu \mathrm{m}$ for a $500 \mathrm{~m}$ baseline, scales inversely \\
\hline Spectral resolution & 25 (possibly 300 ) for exo-planets; 300 for general astrophysics \\
\hline Field of regard & $\begin{array}{l}\text { Annular region between } 46^{\circ} \text { and } 83^{\circ} \text { from anti-sun direction } \\
\text { at a date, } 99 \% \text { over one year }\end{array}$ \\
\hline Target stars & F, G, K, M, at least 150 (10 exo-zodis) or 220 (3 exo-zodi) \\
\hline Mission duration & 5 years baseline, extendable to 10 years \\
\hline Mission profile & Nominal 2 years detection, 3 years spectroscopy, flexible \\
\hline Orbit & L2 halo orbit \\
\hline Formation flying & Radio frequency and laser controlled \\
\hline Station keeping & Field effect electric propulsion (FEEP) or cold gas \\
\hline Launch vehicle & Single Ariane 5 ECA or 2 Soyuz-ST/Fregat \\
\hline
\end{tabular}

additional noise arises from imperfections in the system such as stellar leakage. In order not to dominate the quantum noise, these imperfections must be very stable, with a flat power spectral density, i.e. white noise, allowing the signal to noise ratio on the planet to increase as the square root of the integration time.

Fig. 6 Different flux sources for an Earth analogue at $10 \mathrm{pc}$

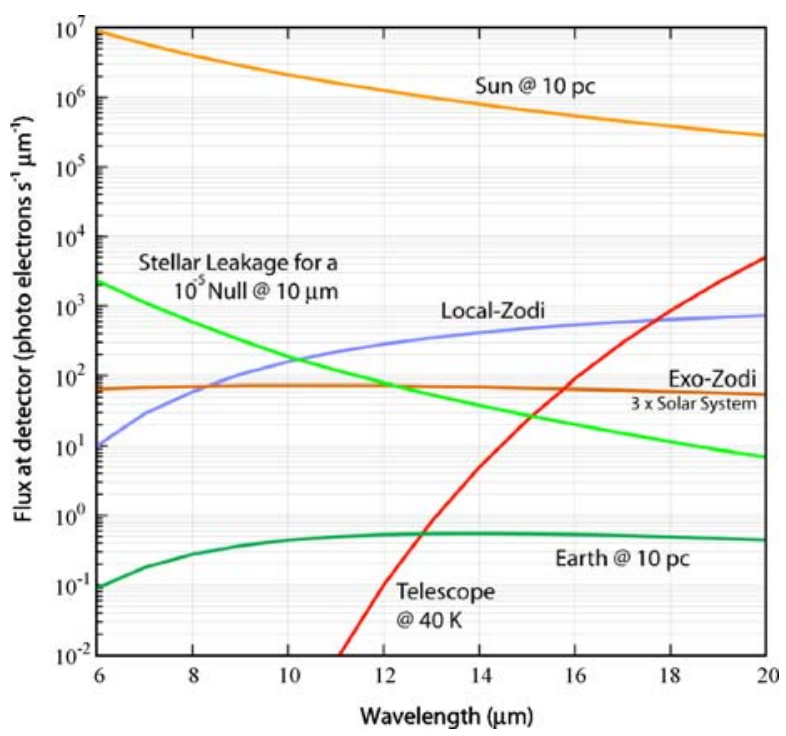




\subsubsection{Instability noise}

To estimate mission performance in a realistic way, we must take into account the possible imperfections of the instrument. In the case of Darwin, the main imperfections result from vibrations and thermal drifts of the spacecraft, which in turn generate small fluctuations in the phase and amplitude of the input light beams. This produces instabilities in the interferometric null, a process similar to speckle noise in a coronagraph. The associated time-variable leakage of stellar photons, called instability noise, is generally not removed by phase chopping $[2,7]$.

Reducing the contribution of instability noise to a harmless level, that is below other unavoidable noises, places strict requirements on configuration control: path length and amplitude variations should be less than $1.5 \mathrm{~nm}$ and $0.1 \%$, respectively.

This is only marginally compliant with state-of-the-art active control. Therefore, two techniques have been proposed and investigated to mitigate the influence of instability noise on mission performance.

The first solution, known as spectral fitting [8], is closely related to the particular arrangement of the X-array, where the nulling baselines are decoupled from the resolution baselines. Stretching the array while keeping the nulling baselines unchanged shrinks the fringe pattern considerably. Because the overall transmission pattern scales as the wavelength, the transmitted planet signal becomes a rapidly oscillating function of wavelength, an effect which can be used to disentangle it from the slowly varying instability noise pattern. The ability of the Emma architecture to adjust the aspect ratio of the X-array is valuable to implement this technique in an optimal way.

The second solution, known as post-nulling calibration, also relies heavily on the geometry of the X-array. The constructive outputs of the pair-wise nulling beam combiners, which contain mostly stellar light, can be used as reference beams to calibrate the final output of the interferometer. Only the stellar component of the output signal produces fringes when combined with these reference beams, and hence the stellar glare can be isolated [6].

These two mitigation techniques represent a decisive advantage of the $\mathrm{X}$-array concept compared to other architectures.

\subsubsection{Search strategy and performance}

Darwin mission performance can be expressed in terms of the number of stars that can be screened for the presence of habitable planets, and the number of follow-up spectroscopic observations that are possible.

The nominal mission is 5 years, with 2 years allocated to detection and 3 years for spectroscopy (possibly reduced by $10 \%$ to $20 \%$ if the imaging capability in constructive mode is implemented-see Section 2.1). About 70\% of mission time is spent collecting data, with the remainder dedicated to overheads, for example, moving the spacecraft to change the interferometer geometry. In order to secure an accurate identification in the search phase, 
we require that the probability for detecting an Earth-like planet in the $\mathrm{HZ}$ at a signal-to-noise ratio (SNR) of 5 be $90 \%$ or larger. We assume that the IR luminosity of planets in the HZ are identical to Earth's, independent of the distance to the host star. Detection is most efficient with a single, optimised array length.

ESA has conducted performance simulations for each star in the target catalogue, using the DarwinSim software to assess the required integration time to reach the required signal to noise ratio (SNR) for detection and spectroscopy. These requirements are a SNR of 5 on the whole band for imaging in nulling mode, and a SNR of 10 from 7.2 to $20 \mu \mathrm{m}$ for $\mathrm{H}_{2} \mathrm{O}, \mathrm{CO}_{2}$ and $\mathrm{O}_{3}$ spectroscopy, using a spectral resolution $\lambda / \Delta \lambda \geq 20$. $^{3}$

The level of exozodiacal emission is an important input parameter to these simulations. The amount of such emission around typical main sequence stars is presently largely unknown. There are successful ongoing programs using the Spitzer spacecraft and Keck-I interferometer to evaluate the zodiacal emission for stars with rather large emission, e.g. young stars, and relate these emissions to stellar parameters. They will be valuable to pre-screen the Darwin targets and avoid object with severe zodiacal emission. The LBT-I interferometer, and a possible future interferometer in Antarctica, will contribute to this effort, in due time before the launch.

Under the assumption that the exozodiacal emission is symmetric around the target star, the chopping process will suppress it, and the exo-zodi will only contribute to the quantum noise. The simulations presented below assume an exozodiacal density of three zodis. ${ }^{4}$

Using an Emma X-array (6:1 configuration) with 2-m diameter telescopes and assuming an optical throughput of $10 \%$ for the interferometer, we estimate that about 200 stars distributed among the four selected spectral types can be screened during the nominal 2-year survey (Table 2). Darwin will thus provide statistically meaningful results on nearby planetary systems. Figure 7 shows that nearby $\mathrm{K}$ and $\mathrm{M}$ dwarfs are the easiest targets in terms of Earth-like planet detection for a given integration time. This is because, the thermal infrared luminosity of a planet in the habitable zone depends only on its size. On the other hand, the stellar luminosity is a strong function of its spectral type. It means that the star/planet contrast varies with spectral type. Compared to the case of the Sun and Earth, this contrast is two times higher for F stars, a factor of three lower for $\mathrm{K}$ stars, and more than an order of magnitude lower for M-dwarfs. However, a special effort is done to observe solar-like $\mathrm{G}$ type stars (50\% of the observing time; Section 3.2) and a significant number of them can be screened and possible terrestrial planets studied (Table 2).

Assuming that each nearby cool dwarf is surrounded by one rocky planet of one Earth radius within its habitable zone, we estimate that only a fraction of

\footnotetext{
${ }^{3}$ The required SNR of 10 for water vapour detection has still to be consolidated. $\mathrm{For}_{\mathrm{CO}_{2}}$ and $\mathrm{O}_{3}$, an SNR of 5 would actually be sufficient for a secure detection.

${ }^{4}$ In practice, exozodiacal densities below 10 times our local zodiacal cloud barely affect the overall shot noise level, while higher densities would significantly increase the required integration times.
} 
Table 2 Number of stars and planets that can be studied

\begin{tabular}{lrrr}
\hline Diameter & $1 \mathrm{~m}$ & $2 \mathrm{~m}$ & $3 \mathrm{~m}$ \\
\hline Screened & 76 & 218 & 405 \\
$\# \mathrm{~F}$ & 5 & 14 & 30 \\
$\# \mathrm{G}$ & 15 & 53 & 100 \\
$\# \mathrm{~K}$ & 20 & 74 & 152 \\
$\# \mathrm{M}$ & 36 & 77 & 123 \\
$\mathrm{CO}_{2}, \mathrm{O}_{3}$ & 17 & 49 & 87 \\
$\# \mathrm{~F}$ & 1 & 2 & 3 \\
$\# \mathrm{G}$ & 4 & 8 & 15 \\
$\# \mathrm{~K}$ & 3 & 12 & 25 \\
$\# \mathrm{M}$ & 9 & 27 & 44 \\
$\mathrm{H}_{2} \mathrm{O}$ & 14 & 24 & 43 \\
$\# \mathrm{~F}$ & 0 & 1 & 1 \\
$\# \mathrm{G}$ & 2 & 4 & 7 \\
$\# \mathrm{~K}$ & 1 & 5 & 10 \\
$\# \mathrm{M}$ & 11 & 14 & 25 \\
\hline
\end{tabular}

the detected planets can be fully characterised (i.e., examined for the presence of $\mathrm{H}_{2} \mathrm{O}, \mathrm{CO}_{2}$ and $\mathrm{O}_{3}$ ) during the subsequent 3-year spectroscopic phase. This number would be doubled or quadrupled for planets with radii 1.5 and 2 times that of the Earth, respectively. A comparable simulation effort at NASA using star count models confirms these predictions.

The diameter of the telescopes has an important incidence on the overall mission performance. With 1-m telescopes, the number of targets screened would be reduced to about 75 , while with 3-m mirrors, almost the complete star catalogue could be surveyed, at constant performance per object.

\subsubsection{Image reconstruction in the nulling mode}

The mission performance estimates in the preceding section are based solely on signal-to-noise ratio, disregarding the details of signal extraction and image reconstruction algorithms. In practice, data processing will be very important,

Fig. 7 Integration time needed for a $5 \sigma$ detection of Earth-sized planets around each of the Darwin candidate targets represented as circles proportional to their physical diameters

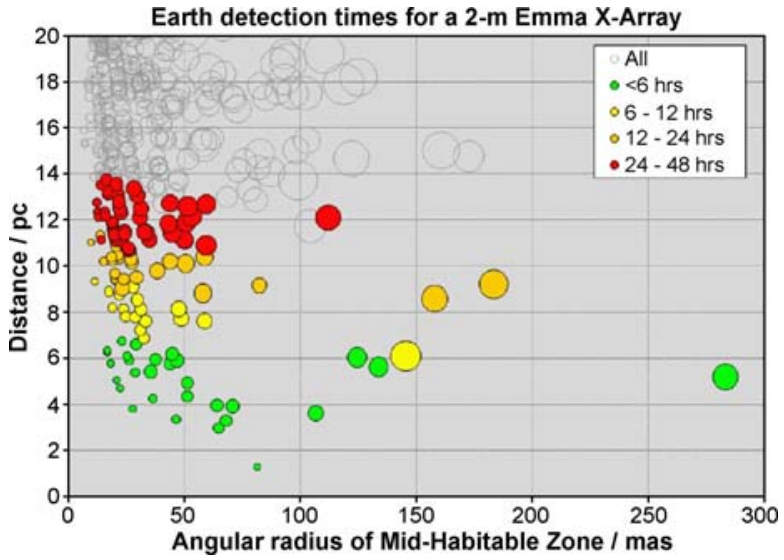




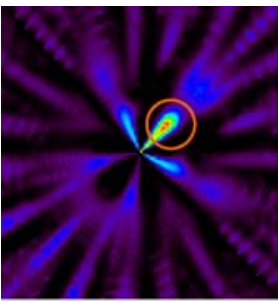

(a) Likelihood map of $1^{\text {st }}$ planet - detected and positioned.

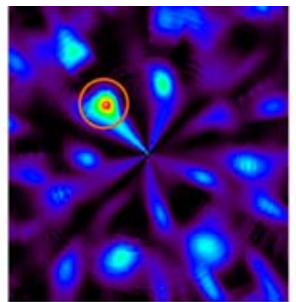

(b) Likelihood map of $2^{\text {nd }}$ planet - detected and positioned

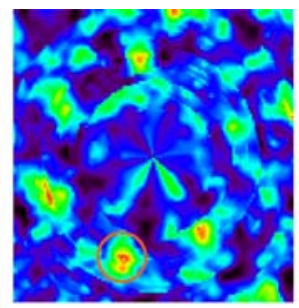

(c) Likelihood map of $3^{\text {rd }}$ planet without regularisation:

ambiguities in position. detected and positioned.

Fig. 8 Likelihood maps of the successive detection of three planets located at $0.64,1.1$ and 1.8 AU of a star. Red indicates a higher probability, black a lower one, white is the highest. The spectral resolution is 15 and $\mathrm{S} / \mathrm{N}$ is 0.33 per spectral element. The third and faintest planet is correctly detected only when the regularisation process is included [13]

especially when several planets are orbiting the same star. For example, accurate orbit determination requires that the emission can be localised and tracked over time, while spectroscopy is only meaningful if the photons can be attributed to the right object. It is very important that Darwin can resolve the emission from the multiple sources that might be present in a stellar system, including planets, lumps in the exozodiacal dust emission, background objects, etc. Image fidelity depends on a high quality PSF, such as that shown for the X-Array in Fig. 3. As mentioned before, this configuration has the major advantage of allowing a separation of the nulling and imaging baselines. Software development for nulling imaging has been initiated on both sides of the Atlantic. In Europe, a Bayesian approach has been chosen. It takes advantage of all the available information for planet reconstruction, for example, incorporating positivity and smoothness of the spectra, a process called "regularisation." Figure 8 illustrates how these constraints can enhance detection performance.

\subsubsection{Visibility measurements with a bright source in the field}

For imaging in the constructive mode, the presence of a bright source in the field makes cophasing the sub-pupils of the interferometer much easier. The nature of the target and the science goal will determine the required instrumentation. We consider three different cases: few visibility measurements with a bright source in the field of view, imaging with a bright source, and imaging without a bright source.

With minimal impact on the nulling recombiner, Darwin can carry out visibility $\left(\mathrm{V}^{2}\right)$ science with JWST-like sensitivity, as long as there is a $K \leq 13$ magnitude source in the field of view to stabilize the array. The modulus of the visibility provides simple size information about the target, for example, its radius assuming spherical morphology. The phase of the visibility gives shape information, such as deviations from spherical symmetry. 

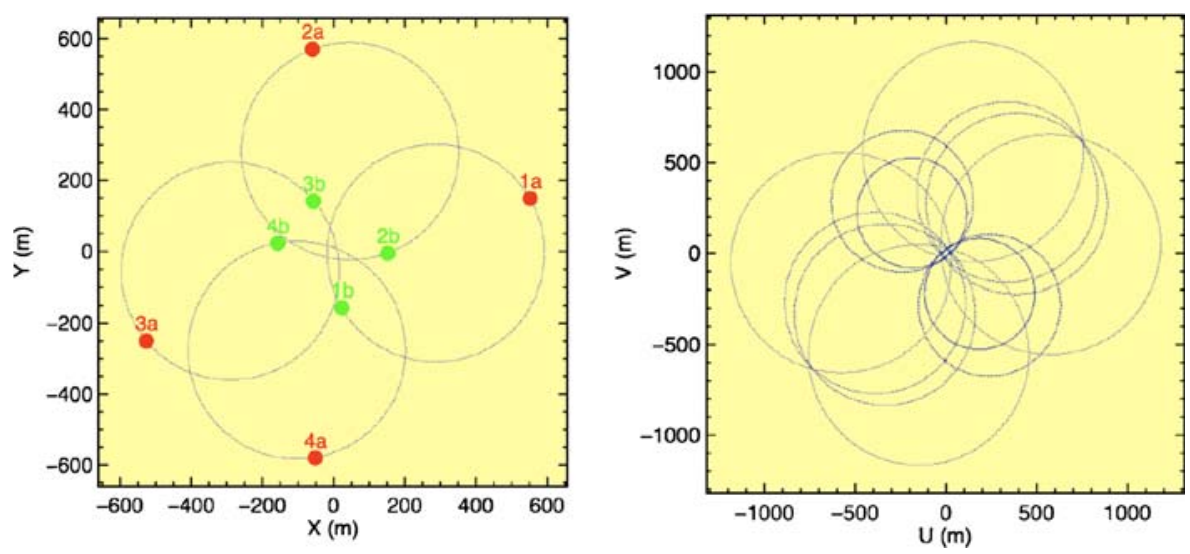

Fig. 9 Possible satellite tracks and UV-coverage for four collectors. The satellites independently move in circles (left) with the hub located at $X=0, Y=0$, continuously varying the dimensions of the array. The positions of the spacecraft are represented at two different epochs (a and $\mathbf{b})$. The resulting filling of the $(u, v)$ plane is already rather good after a single tour of spacecrafts $(r i g h t)$

If the target spectrum is smooth a few visibility measurements can be obtained rapidly, because Darwin can work simultaneously at several wavelengths (see below). The baseline recombiner could perform such measurements with very modest modification, and hence, with minimum impact on the cost of the mission. Therefore, a capability for basic visibility measurement should be implemented. Unfortunately, a limited number of $\mathrm{V}^{2}$ observations provide useful information for only a limited number of targets.

\subsubsection{Aperture synthesis for targets with a bright source}

To obtain a fully reconstructed image, the $(u, v)$ plane must be filled by moving the array. A significant gain in efficiency can be realized if the spectrum of the target is smooth over the operating band of the instrument. The shorter wavelengths sample higher spatial frequencies, and the longer wavelengths lower spatial frequencies, all at the same array spacing. Figure 9 show an example of $(u, v)$ coverage when the individual spacecrafts have circular trajectories. A $100 \times 100$ image could be obtained with a few hundreds of positions, rather than the 10,000 positions required for a full spatial and spectral reconstruction. Table 3 lists the requirements for this mode.

Table 3 Aperture synthesis imaging requirements for Darwin

\begin{tabular}{lll}
\hline & Requirement & Goal \\
\hline Maximum baseline $(\mathrm{m})$ & 300 & 500 \\
Minimum baseline $(\mathrm{m})$ & 20 & 10 \\
Field of view (resolution elements) & $100^{2}$ & $300^{2}$ \\
Dynamic range & $1: 100$ & $1: 1,000$ \\
Spectral range $(\mu \mathrm{m})$ & $6-20$ & $4-30$ \\
Spectral resolution & 300 & 3,000 \\
\hline
\end{tabular}


Sensitivity The $5 \sigma, 1 \mathrm{~h}$, point source sensitivities for Darwin in $20 \%$ wide bands centred at $8,10,13$ and $17 \mu \mathrm{m}$ are approximately $0.1,0.25,0.5$ and $0.8 \mu \mathrm{Jy}$, respectively. These sensitivities are comparable to those of JWST.

Angular resolution The maximum foreseen baselines are $500 \mathrm{~m}$, corresponding to a spatial resolution of 5 mas at $10 \mu \mathrm{m}$.

Co-phasing With a stability time scale of $10 \mathrm{~s}$ for the array (Alcatel Study, 2000), the sensitivity limit for self-fringe-tracking is about $10 \mathrm{mJy}$ at $10 \mu \mathrm{m}$ in a 0.5 arcsec aperture. This performance gives access to virtually all of the sources in the Spitzer SWIRE survey.

\subsubsection{Aperture synthesis for targets with no bright source}

For targets with no bright source in the field, the preferred option for cophasing is the use of a nearby off-axis bright reference star $(K \leq 13)$. One way of doing this is to feed the K-band light of this star along with the 6-18 $\mu \mathrm{m}$ light of the target to the beam combiner satellite, a so-called dual field configuration. Another option is to make the interferometer optically rigid using kilometric optical gyros. These devices can maintain the phasing of the array between pointings at a reference star and the target field.

Clearly, this additional instrumentation may be much more demanding and expensive. A decision whether or not to add this capability to Darwin will depend on an analysis undertaken during the study phase and available fundings.

\section{Science operations and archiving}

\subsection{Data science operations architecture and share of responsibilities}

The Science Operations Center (SOC) will be responsible for science mission planning, data processing, and data product distribution to the Darwin science team and the wider scientific community. Because the data acquisition and calibration requirements are very different for the planetary (nulling) and general astrophysics (imaging) missions, options for the SOC beyond ESOC need to be considered. Computer networking and remote presence through videoconferencing will play a central role, allowing responsibilities to be spread among a variety of network-nodes at several institutes throughout Europe.

\subsection{Archive approach}

The site for active and legacy archives is to be determined. The archive should include values of the nulling transmission, visibilities, and reduced image data, including the accompanying calibration files. A quick-look facility will allow 
rapid assessment and review of the data. Compared to other contemporary missions, Darwin's data volume will be relatively modest and should present no storage challenges.

\subsection{Propriety data policy}

Although the detailed rules of data access are still to be determined, we anticipate that there may be different policies for the primary science and general astrophysics programs. Specifically, the baseline mission (nulling interferometry) will be conducted by ESA in cooperation with a dedicated team of Darwin scientists. Data rights would then follow guidelines adopted by ESA for missions similar in character (e.g., GAIA). In general, the science team is obliged to reduce the data and make the results public within a stipulated time. A peer-review process will almost certainly determine the general astrophysics targets. Following a call for Open Time observations, ESA will accept proposals from a Lead Scientist, who will act as the contact point between the Agency and the proposing community. In this case, the commonly adopted proprietary period is one year from the time of data release.

\section{Technology and mission roadmap for Darwin}

\subsection{Darwin's technology roadmap}

\subsubsection{Essential technology developments for Darwin}

The pre-assessment study of Darwin by Alcatel in 2000, and the assessment study by TAS and Astrium in 2006 determined that there are no technology show stoppers for this ambitious mission. However, two key areas were identified that require focused attention and resources:

- Formation flying of several spacecrafts with relative position control of a few centimetres

- The feasibility of nulling interferometry in the 6-20 $\mu \mathrm{m}$ range. Based on the expected star/planet contrast $\left(1.5 \times 10^{-7}\right.$ at $10 \mu \mathrm{m}$ and $10^{-6}$ at $18 \mu \mathrm{m}$ for an Sun-Earth analogue) and on evaluations of instability noise (Section 3.3.2), the common conclusion of the industrial studies is that the null depth must be $10^{-5}$ on average, and that it must be sufficiently stable on the timescale of days so that the signal to noise ratio improves essentially as the square root of time. This stability requirement translates into tight instrument control specifications, which can however be relaxed by means of the two instability noise mitigation techniques presented in Section 3.3.2. A thorough evaluation of the these techniques and of the resulting instrumental stability requirements will be a key component of the technology development programme. 


\subsubsection{Current status of technology development}

Europe has devoted considerable resources, both intellectual and financial, to these technological issues since the initial Alcatel study. ESA has invested approximately $20 \mathrm{M} €$ since 2000 , with a significant ramp-up in the last 2 years. Several tens of Technology Research Programs (TRPs) have been issued. NASA has run a parallel program in the USA. Most of the key technologies have been addressed and significant progress achieved.

In the area of formation flying (FF), the TRPs "interferometer constellation control" (ICC1 and ICC2) have developed nonlinear, high fidelity navigation simulators. Algorithms for interferometer constellation deployment at L2 have also been demonstrated. In the USA, analogous simulations and a 2D robotic breadboard (Fig. 10) have shown the feasibility of formation flying. Finally, with the PRISMA mission being prepared for launch next year (Section 5.2), formation flying is approaching technology readiness level (TRL 5/6).

The investment in nulling interferometry research over the past 7 years has brought the technology to TRL 4 . The flight requirement is a null depth of $10^{-5}$ in the 6-20 $\mu \mathrm{m}$ domain. In Europe and at the JPL monochromatic experiments using IR lasers at 3.4 and $10.6 \mu \mathrm{m}$, have yielded nulls equal to or significantly better than $10^{-5}$ (Fig. 11). Broadband experiments have achieved nulls of $1.2 \times$ $10^{-5}$ for $32 \%$ bandwidth at $10 \mu \mathrm{m}$, closely approaching the flight requirement [16]. Clearly, the technology of nulling interferometry is nearing maturity, although it has not yet been demonstrated over the full Darwin bandwidth with the required depth and stability. These results give us confidence that the mission goals will be met with continued effort and investment.

Additional key technological developments in recent years include:

- Selection of the baseline interferometer configuration. Significant effort in this area since 2000, backed by independent studies in the USA and Europe, has identified the non-planar Emma X-Array as the optimal choice (see Section 3.2);

Fig. 10 The two robots of the formation control testbed at JPL. Each robot carries a mobile instrument platform (shown tilted), as well as canisters of compressed air to float the robot on a polished metal floor. The testbed has completed its functional testing and should achieve operational testing in 2007

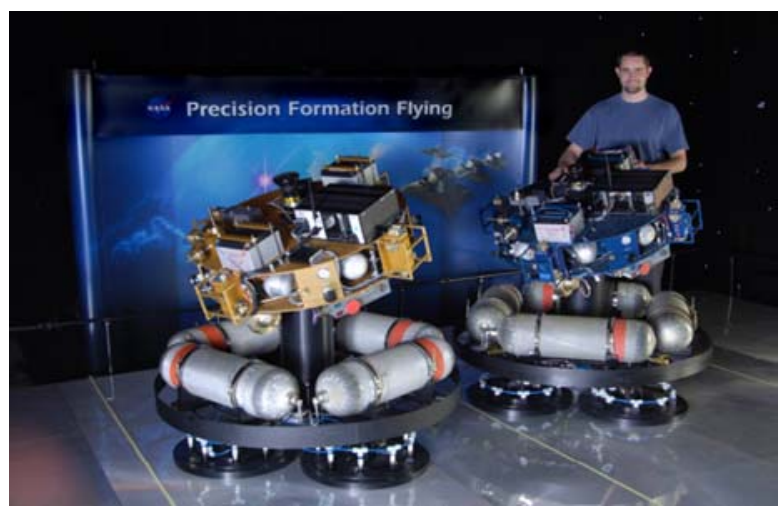



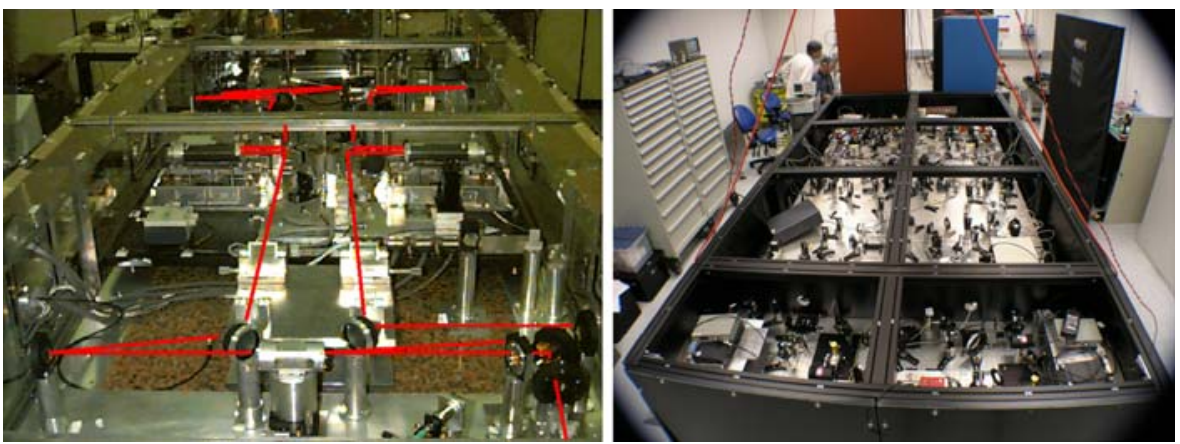

Fig. 11 Two nulling interferometer testbeds. Left, the experiment by Ollivier et al. [14] at IAS, Orsay. The superimposed red lines indicate the optical paths. An input beam is divided into two parts to simulate the light coming from two different spacecrafts. The beams recombine in destructive or nulling interference mode. Right, the planet detection testbed at JPL, which simulates a bright star and a faint planet. The planetary signal can be extracted from the global flux when the contrast ratio is below two million

- Achromatic phase shifters (APS), which allow broadband destructive interference between beams, have reached TRL 4. A comparative study currently running in Europe should identify the preferred approach;

- Space-qualified delay lines to balance the different optical paths to nanometre accuracy have been demonstrated to TRL 5. A breadboard at TNO-TPD has achieved this performance at $40 \mathrm{~K}$ and may be included as a test payload in the PROBA 3 space mission (Section 5.2);

- Single mode fibres, or integrated optics modal filters that enable broadband nulling are now at TRL 4. Chalcogenide fibres have demonstrated the required performance of $40 \%$ throughput and $30 \mathrm{~dB}$ rejection of higher order spatial modes in the $6-12 \mu \mathrm{m}$ band. On going work is emphasizing silver halide single-mode filters, which will operate in the 12-20 $\mu \mathrm{m}$ band. Photonic crystal fibres that can cover the whole spectral domain in a single optical channel are considered;

- Detector arrays with appropriate read noise and dark current are at TRL 5/6. The Si:As impurity band conductor (IBC) arrays developed for JWST appear to be fully compliant with Darwin requirements. A reduced-size version of the JWST $1,024 \times 1,024$ detector, e.g. $512 \times 8(300 \times 300$ for the general astrophysics program), could be read out at the required rate with a dissipation of a few tens to hundreds of $\mu \mathrm{W}$. These devices exhibit high quantum efficiency $(80 \%)$, low read noise $\left(19 \mathrm{e}^{-}\right)$, and minimal dark current $\left(0.03 \mathrm{e}^{-/ \mathrm{s}}\right.$ at $\left.6.7 \mathrm{~K}\right)$. Such performance permits sensitive observations, even at moderately high spectral resolution $(\operatorname{Res}=300)$;

- Low vibration Cryo-coolers for the detector system are now at TRL 4. A European TRP has led to a prototype absorption cooler providing $5 \mathrm{~mW}$ of cooling power at $4.5 \mathrm{~K}$. JPL scientists have demonstrated a system with $30 \mathrm{~mW}$ of cooling at $6 \mathrm{~K}$. 


\subsubsection{Future plans}

The message from the last decade of Darwin technology development is clear: if the research and technology effort that has been pursued in both Europe and the USA continues vigorously, Darwin's technology will reach TRL 5/6 by 2010, allowing it to be selected as ESA's first L mission for launch in 2018-2020.

In addition to the European and American teams, the Japanese space agency (JAXA) has expressed interest in the mission and in participating in the technology effort. Japan has considerable expertise in several key fields, including cryogenics with the AKARI mission, and mirror engineering.

\subsection{Precursor missions}

\subsubsection{Exoplanet discovery and statistics}

COROT (in operation) COROT is a CNES led mission that searches for planetary transits (Fig. 12). It was launched at the end of 2006 and commissioning is running very successfully (a transit with excellent quality light curve has been quoted). It has a $27 \mathrm{~cm}$ off-axis telescope and will observe five or more fields with about 12,000 stars each for a period of 5 months. COROT can detect planets with $R_{\mathrm{pl}} \geq 2 R_{\text {Earth }}$ and orbital periods $\leq 50$ days. As early as $2008-$ 2009, COROT should provide statistics on these objects and, by extrapolation, information on the abundance of terrestrial planets in the HZ.

Kepler (under implementation) Scheduled for 2008-2009, Kepler will detect terrestrial and larger planets near the $\mathrm{HZ}$ of stars with a wide variety of spectral types [5] (Fig. 13). Its $0.95 \mathrm{~m}$ diameter telescope, pointed continuously at a single field, will monitor about 100,000 main-sequence stars located a few hundreds parsecs away with the precision to detect Earth-sized planet transits. Over its 4-year lifetime, Kepler should provide the statistical abundance of the

Fig. 12 The COROT mission can detect hot big earths

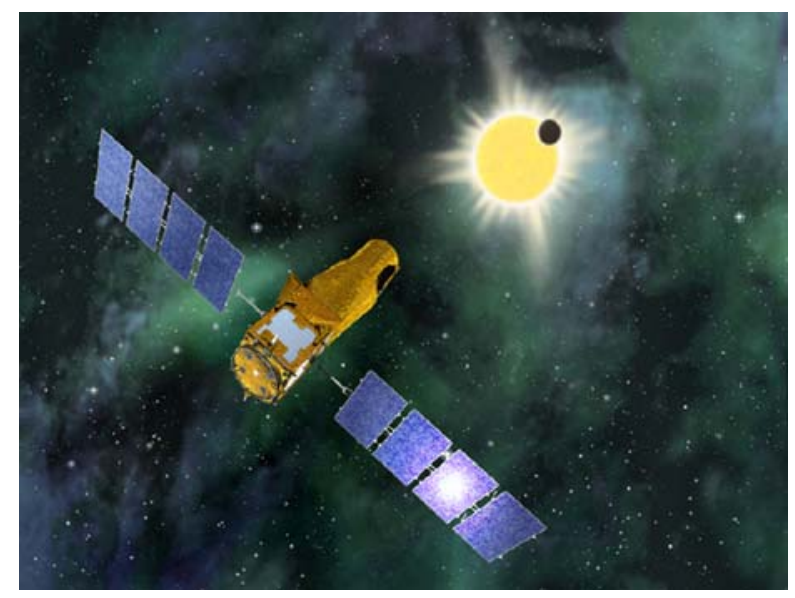


Fig. 13 Kepler can detect Earth-like planets

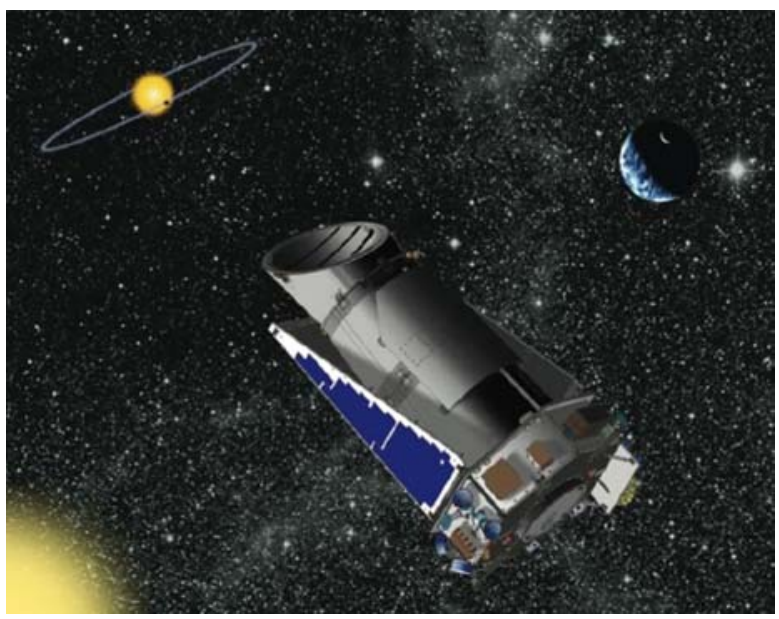

type of terrestrial planets that Darwin aims to characterize. This information will be valuable for Darwin mission planning.

\subsubsection{Formation flying}

The Darwin interferometer relies on formation flying (FF) technology to control the four collector spacecrafts and one beam combiner. Section 3 describes how this strategy offers significant advantages. As with any new approach, however, FF should be validated in space. Europe has initiated several precursor missions:

PRISMA (approved) PRISMA is a Swedish-led technology mission, which intends to demonstrate FF and Rendez-vous technologies bringing them to TRL 8/9 (Fig. 14). The Swedish Space Corporation is leading this effort, which is funded by Sweden, Germany, Denmark, France and Alcatel. The mission comprises two spacecraft and should be launched in autumn 2008 into a low, Sun-synchronous orbit (600-1,000 km), with a mission lifetime of about 8 months. The main objectives are to carry out technological flight demonstrations and manoeuvring experiments, including guidance, navigation, control, and sensor techniques [15]. The positioning of the spacecraft relies on an Alcatel relative GPS technology, which should have an accuracy of $\sim 10 \mathrm{~cm}$. For intra-satellite distances less than $6 \mathrm{~m}$, additional optical metrology should improve this accuracy.

PROBA 3 The PROBA-3 mission is the next logical step after PRISMA. This mission builds on PRISMA's achievements, in addition to RF metrology, will demonstrate optical metrology sensors to TRL 8/9, providing $30 \mu \mathrm{m}$ relative positioning accuracy. PROBA 3 could be launched in 2010 , but it is not yet fully funded. This mission is not essential for Darwin, but we favour its completion, because it would provide further in-space demonstration of FF technologies. 
Fig. 14 Artist's rendition of PRISMA. The larger of the two spacecraft carries most of the equipment and orbits around the smaller vehicle

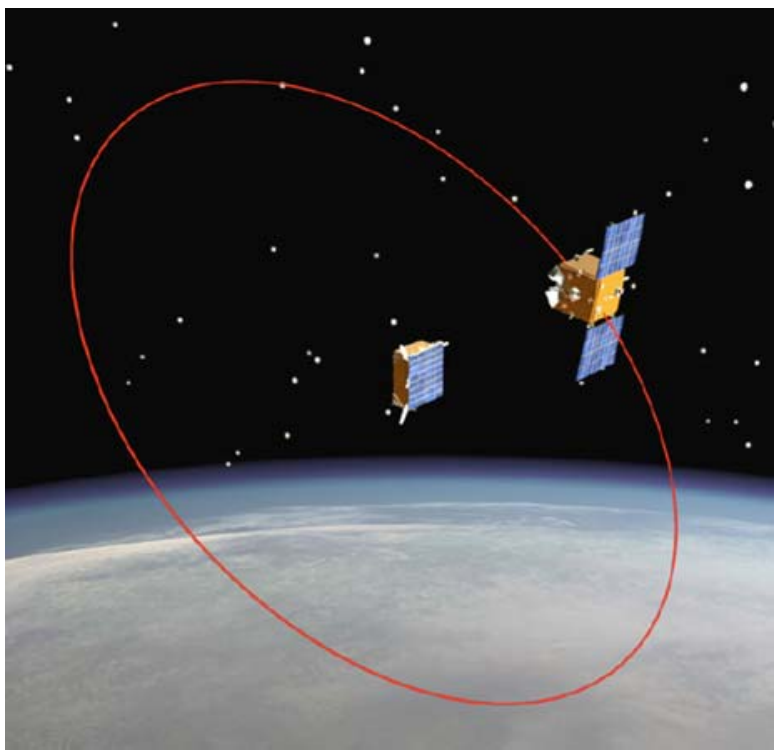

Pegase/PERSEE Pegase is a single Bracewell interferometer that was proposed in the framework of the 2004 call by CNES for its formation flying demonstrator mission. The main scientific goal of Pegase is the high-angular resolution study of extrasolar giant planets at near-infrared wavelengths $(2.5$ to $5 \mu \mathrm{m})$. The mission could be extended to the study of brown dwarfs, circumstellar disks and dust tori around active galactic nuclei (AGN). CNES performed a Phase 0 study, but the mission was not selected for budgetary reasons. The construction of a mission breadboard, called PERSEE, is under consideration to increase our understanding of nulling interferometers. Note that, in order to ensure knowledge transfer to ESA, Pegase is being proposed as an M mission within the Cosmic Vision programme. An attractive possibility would be a merger with PROBA 3, with additional European and possibly international participation. This would allow the inclusion of stellar interferometry into the mission.

\section{Cost plan and international cooperation}

\subsection{Cost estimate}

Darwin is proposed as an L mission. Darwin will be one of the most ambitious missions that ESA has ever undertaken, and we emphasize that a phase A study is needed to derive an accurate cost estimate. Here, we present a preliminary estimate, based on information from Alcatel (now Thales Alenia Space, TAS), Astrium, and a recent study of the Emma X-array by JPL. 
The introduction of the Emma concept $[4,17]$ provides a major simplification of the instrument, eliminating all deployable components except the antennas. The optics of the collecting spacecrafts (CS) is reduced to a single mirror (Section 3.2). Note that the CS cost scales as a function of the mirror diameter, because it is the main component of the spacecraft.

We present the costing for a $2 \mathrm{~m}$ version as a baseline, but also show the cost and performance trade for Darwin with mirrors of diameter 1.5 and $1.0 \mathrm{~m}$. The corresponding numbers of stars screened and planets studied change, but at constant quality per object (Section 3.3.3). These numbers appear in Table 4 below.

Further cost savings may be achievable in the design of the beam combiner spacecraft (BCS). For instance, the current baseline design requires two distinct optical paths, because of the limited spectral range of existing singlemode fibres. However in the near future, Photonic Crystal fibres may allow operation over the entire 6-18 $\mu \mathrm{m}$ range with a single optical path.

Baseline cost items for the Emma X-array configuration with $2 \mathrm{~m}$ collecting mirrors:

- Flight elements. We use the JPL estimate of spacecraft masses plus a $20 \%$ margin, and apply a mean cost of $220 \mathrm{k} €$ per payload $\mathrm{kg}$, which includes $15 \%$ contingency. These values are typical for scientific payloads (Earth and astronomical observations) as well as for telecommunication satellites, (information supplied by a European prime contractor). Total, $845 \mathrm{M} €$.

- Launcher. Ariane V ECA, 6.6 tons deliverable to L2: $125 \mathrm{M} €(\mathrm{CV}$ Annex 4).

- Ground segment (5 years operations). Considering the volume of communications, a prime contractor estimates the ground segment cost at $55 \mathrm{M} €$, which seems conservative when compared to that of GAIA (48 M€).

- Pre-implementation and Space Agency internal costs. We apply $1 \%$ and $11 \%$ of the total, respectively, as required in CV Annex 4.

- Contingency. This is already included: $20 \%$ on mass plus $15 \%$ on cost per kilogram.

Table 4 Estimated total cost and science performance for Darwin with different collector spacecraft mirror diameters

\begin{tabular}{llllll}
\hline $\begin{array}{l}\text { Diam. } \\
(\mathrm{m})\end{array}$ & $\begin{array}{l}\mathrm{M} \\
(\mathrm{kg})\end{array}$ & $\begin{array}{l}\text { Total cost } \\
(\mathrm{M} €)\end{array}$ & $\begin{array}{l}\text { Screened } \\
\text { stars }\end{array}$ & $\begin{array}{l}\text { Planets } \\
\mathrm{O}_{3}, \mathrm{CO}_{2} \mathrm{sp} .\end{array}$ & $\begin{array}{l}\text { Planets } \\
\mathrm{H}_{2} \mathrm{O} \text { sp. }\end{array}$ \\
\hline 2.0 & 3,830 & 1,200 & 218 & 49 & $(24)$ \\
1.5 & 2,960 & 950 & 142 & 32 & $(18)$ \\
1.0 & 2,290 & 800 & 76 & 17 & $(14)$ \\
\hline
\end{tabular}

Row with values in italics indicates the baseline choice

${ }^{\text {a }}$ The method for detecting $\mathrm{H}_{2} \mathrm{O}$ is presently under examination, as is the number of planets for which this molecule can be searched for 
The total cost for $2 \mathrm{~m}$ collectors is then: $845+125+55+12+128=1165 \mathrm{M} €$ $\approx 1,200 \mathrm{M} €$.

Table 4 presents cost and performance estimates for different CS mirror sizes, using a conservative scaling dependence of the collecting mirror mass with their diameter, $\mathrm{M} \propto D^{2}$.

\subsection{International cooperation}

Darwin science has worldwide appeal. At present, both NASA and the Japan space agency, $J A X A$, have indicated their interest in the mission and their willingness to participate in the study phase (letters of commitment has been sent). Their letters also indicate a possible participation in the construction and operation phases. Contacts with other agencies are being cultivated.

Open Access This article is distributed under the terms of the Creative Commons Attribution Noncommercial License which permits any noncommercial use, distribution, and reproduction in any medium, provided the original author(s) and source are credited.

\section{References}

1. Bracewell, R.N.: Detecting non-solar planets by spinning infrared interferometry. Nature 274, 780-781 (1978). doi:10.1038/274780a0

2. Chazelas, B.: Instrumental stability requirements for exoplanet detection with a nulling interferometer: variability noise as a central issue. Appl. Opt. 45, 984 (2006). doi:10.1364/AO. 45.000984

3. Kaltenegger, L., Eiroa, C., Fridlund, M.: Target star catalogue for Darwin, Nearby Stellar sample for a search for terrestrial planets. Astron. Astrophys. (2008, submitted)

4. Karlson, A.: Three telescope nuller based on multibeam injection into single-mode waveguide. Proc. SPIE 5491, 831 (2004). doi:10.1117/12.550307

5. Koch, D., Borucki, W., Lissauer, J.J., Dunham, E.W., Jenkins, J.: The Kepler Mission, a search for habitable planets: concept, capabilities and strengths. Bull. AAS 30, 1058 (1998)

6. Lane, B.F., Muterspaugh, M.W., Shao, M.: Calibrating an interferometric null. Astrophys. J. 648, 1276 (2006). doi:10.1086/506020

7. Lay, O.P.: Systematic errors in nulling interferometers. Appl. Opt. 43, 6100 (2004). doi:10.1364/AO.43.006100

8. Lay, O.P.: Removing instability noise in nulling interferometers. Proc. SPIE 6268, 62681A (2006)

9. Lay, O.P., Dubovitsky, S.: Nulling interferometers: the importance of systematic errors and the X-Array configuration. SPIE 5491, 874 (2004)

10. Léger, A., Mariotti, J.M., Mennesson, B., Ollivier, M., Puget, J.L., Rouan, D., et al.: Darwin proposal to ESA Horizon 2000+ Missions (1993)

11. Léger, A., Mariotti, J.M., Mennesson, B., Ollivier, M., Puget, J.L., Rouan, D., et al.: Could we search for primitive life on extrasolar planets in the near future? The DARWIN project. Icarus 123, 249-255 (1996). doi:10.1006/icar.1996.0155

12. Mennesson, B., Léger, A., Ollivier, M.: Direct detection and characterization of extrasolar planets: the Mariotti space interferometer. Icarus 178, 570-588 (2005). doi:10.1016/j. icarus.2005.05.012

13. Mugnier, L.M., Thiébaut, E., Belu, A.: In: 2006 Data processing in nulling interferometry: case of the Darwin mission, in astronomy with high contrast imaging III. EAS Publications Series, EDP Sciences, Les Ulis, France (2006) 
14. Ollivier, M., Mariotti, J.-M., Léger, A., Sekulic, P., Brunaud, J., Michel, G.: Interferometric coronography for the $D A R W I N$ space mission-laboratory demonstration experiment. A\&A 370, 1128-1136 (2001)

15. Persson, S., Jacobsson, B.: PRISMA - Swedish in-orbit testbed for rendezvous and formation flying. In: Proceedings of the 57th IAC/IAF/IAA (International Astronautical Congress), Valencia, Spain, Oct. 2-6, IAC-06-D1.2.02 (2006)

16. Peters: http://planetquest.jpl.nasa.gov/TPF-I/ (2007)

17. Thalès: Alenia Space, Darwin Assessment Study to ESA (2006) 